\title{
NULL SPACES AND RANGES OF POLYNOMIALS OF OPERATORS
}

\author{
MANUEL GONZÁLEZ
}

\begin{abstract}
We give an elementary proof of the fact that given two polynomials $P, Q$ without common zeros and a linear operator $A$, the operators $P(A)$ and $Q(A)$ verify some properties equivalent to the pair $(P(A), Q(A))$ being non-singular in the sense of J.L. Taylor. From these properties we derive expressions for the range and null space of $P(A)$ and spectral mapping theorems for polynomials of continuous (or closed) operators in Banach spaces.
\end{abstract}

Let $X$ be a complex linear space, $A \in L(X)$ a linear map on $X$. If $\lambda, \mu \epsilon$ $\in \mathcal{C}, \lambda \neq \mu$, then

$$
(\lambda-A) x=(\lambda-\mu) x+(\mu-A) x \quad \text { for each } \quad x \in X
$$

From this simple equality it follows a big amount of information about the relations between the null spaces and ranges of $S=\lambda-A$ and $T=\mu-A$. In fact we have:

$$
\begin{array}{ll}
\text { (a) } & N(S) \cap N(T)=\{0\} \\
\text { (b) } & R(S)+R(T)=X \\
\text { (c) } & T[N(S)]=N(S) ; S[N(T)]=N(T) \\
\text { (d) } & R(S T)=R(S) \cap R(T) \\
\text { (e) } & N(S T)=N(S) \oplus N(T)
\end{array}
$$

$(a),(b)$ and $(c)$ follow immediatly from $\left(^{*}\right)$, and as $S T=T S$ we have $R(S T) \subset R(S) \cap R(T)$; but if $z \in R(S) \cap R(T)$, then $z=(\lambda-A) x=(\mu-A) y$. Using $\left(^{*}\right)$ we obtain $y=(\mu-\lambda)^{-1}(\lambda-A)(x-y)$.

Hence $z=(\mu-A)(\lambda-A)\left\{(\mu-\lambda)^{-1}(x-y)\right] \in R(S T)$. Finally by (c) we have $T^{-1}[N(S)]=N(S)+N(T)$. Then taking account of $(a)$ we conclude $N(S T)=N(S) \oplus N(T)$. 
In this note we shall prove that given $P(z)$ and $Q(z)$ polynomials without common zeros, the operators $S=P(A)$ and $T=Q(A)$ also verify the five properties above. As a corollary we obtain expressions for $R[P(A)]$ and $N[P(A)]$ as functions of the ranges and ntul spaces of $(\lambda-A)^{n}, \lambda$ zero of $P(z)$ of order $n$, as is obtained in [4] with a different proof, and some other interesting consequences related with the spectral mapping theorem for polynomials as given in [1].

We start proving an auxiliary result.

Proposition. Let $S, T \in L(X)$ such that $S T=T S$.

(i) $S$ and $T$ verify (c) and (d) if and only if given $y, z \in X$ such that $S y=T z$, there exists $x \in X$ with $y=T x$ and $z=S x$.

(ii) If $S$ and $T$ verify (a) and (c), then they verify (e).

\section{Proof:}

(i) Suppose $S$ and $T$ verify (c) and (d), and $S y=T z=u$. As $u \in$ $\in R(S) \cap R(T)=R(S T)$, there exist $v$ such that $u=S T v=T S v$. Then $T v-y \in N(S)$ and $S v-z \in N(T)$. Using (d) we can choose $f \in N(S)$ and $g \in N(T)$ such that $T v-y=T f$ and $S v-z=S g$. Taking $x=v-f-g$ we have $T x=y$ and $S x=z$.

Conversely, let $S$ and $T$ verifying the second part of (i). We have $R(S T) \subset$ $\subset R(S) \cap R(T)$. Let $v \in R(S) \cap R(T)$. Then $v=T y=S z$ for some $y, z$. Taking $x$ such that $z=T x$ we obtain $v=S T x \in R(S T)$. Moreover $T[N(S)] \subset N(S)$. Let $y \in N(S) . T 0=S y=0$ and then there exist $x$ such that $S x=0$ and $T x=y$. Hence $T[N(S)]=N(S)$. Analogously $S[N(T)]=N(T)$.

(ii) As $T[N(S)]=N(S)$, taking account of (a) we conclude $N(S T)=$ $=T^{-1}[N(S)]=N(S) \oplus N(T)$.

Definition. Let $S, T \in L(X)$ a pair of commuting operators. If $S$ and $T$ verify (a), (b), (c) and (d) (and then also (e)), we shall say that the pair $(S, T)$ is non-singular.

Note that the above definition coincides with the non-singularity in the sense of J.L. Taylor [3], used to define a joint spectrum for two commuting operators. We don't follow that way. The following theorem is the central result in this note as it will allow us to pass from an operator to their polinomials and vice versa.

Theorem. Let $A, B, T \in L(X)$ commuting operators; $S=A B .(S, T)$ is non-singular if and only if $(A, T)$ and $(B, T)$ are non-singular.

Proof: Suppose $(S, T)$ non-singular. As $N(A)+N(B) \subset N(S)$ and $R(S) \subset$ $C R(A) \cap R(B)$ we obtain (a) and (b) for $(A, T)$ and $(B, T)$ from these properties for $(S, T)$. Let $y, z \in X$ such that $T y=A z$. By (b) there exist $u, v \in X$ such that $z=B u+T v$ and so $A z=A B u+A T v=S u+T A v$. Then $S u=T(y-A v)$. 
Let $w$ such that $T w=u$ and $S w=y-A v$. Taking $x=B w+v$ we have $T x=T B w+T v=B u+T v=z$, and $A x=A B w+A v=S w+A v=y$. Using the above proposition we conclude that $(A, T)$ is non-singular. The proof for $(B, T)$ is the same.

Suppose now $(A, T)$ and $(B, T)$ non-singular. If $x \in N(S) \cap N(T)$, then $A x \in N(B) \cap N(T)=\{0\}$, and so $x \in N(A) \cap N(T)$. Hence $x=0$. Let $x \in X$. $x=T y+A z$ for some $y, z$ in $X$, and $z=T u+B v$ for some $u, v$ in $X$. Then $x=T y+A T u+A B v=T(y+A u)+S v$. Hence $R(S)+R(T)=X$. Finally let $S y=T z$. As $A B y=T z$ there exist $v$ in $X$ such that $A v=z$ and $T v=B y$. Now we can take $x$ in $X$ such that $T x=y$ and $B x=v$; and so $S x=A v=z$. Hence we conclude that $(S, T)$ is non-singular.

Corollary. Let $A \in L(X)$ and $P(z)$ and $Q(z)$ polynomials without common zeros. Then we have

(i) $(P(A), Q(A))$ is non-singular.

for $i \neq j$ then

(ii) If $P(z)=\left(\lambda_{1}-z\right)^{m_{1}} \ldots\left(\lambda_{k}-z\right)^{m_{k}}$ with $m_{i}$ integers and $\lambda_{i} \neq \lambda_{3}$

$$
\begin{aligned}
& R[P(A)]=R\left[\left(\lambda_{1}-A\right)^{m_{1}}\right] \cap \cdots \cap R\left[\left(\lambda_{k}-A\right)^{m_{k}}\right] \\
& N[P(A)]=N\left[\left(\lambda_{I}-A\right)^{m_{1}}\right] \oplus \cdots \oplus N\left[\left(\lambda_{k}-A\right)^{m_{k}}\right]
\end{aligned}
$$

(iii) If $X$ is a Banach space and $A$ is continuous, then $R[P(A)]$ closed if and only if $R\left[\left(\lambda_{i}-A\right)^{m_{i}}\right]$ closed; $i=1, \ldots, k$.

$R[P(A)]$ dense if and only if $R\left[\left(\lambda_{i}-A\right)^{m_{i}}\right]$ dense; $i=1, \ldots, k$.

Proof:

(i) If $P(z)=\left(\lambda_{1}-z\right)^{m_{1}} \ldots\left(\lambda_{k}-z\right)^{m_{k}}$ and $Q(z)=\left(\mu_{1}-z\right)^{n_{1}} \ldots$ $\ldots\left(\mu_{s}-z\right)^{n_{0}}$ then $\left(\lambda_{i}-A, \mu_{j}-A\right)$ is non-singular for every $i$ and $j$, and the result follows immediately from the theorem.

(ii) If $\lambda_{i} \neq \lambda_{j}$ then $\left(\left(\lambda_{i}-A\right)^{m_{i}},\left(\lambda_{j}-A\right)^{m_{j}}\right)$ is non-singular. The results now are consequence of (d) and (e) taking account of the proposition.

(iii) If $R\left[\left(\lambda_{i}-A\right)^{m_{i}}\right], i=1, \ldots, k$ are closed then from (ii) it is evident that $R[P(A)]$ is closed. On the other hand, making $B=\left(\lambda_{1}-A\right)^{m_{1}} \ldots$ $\ldots\left(\lambda_{k-1}-A\right)^{m_{k-1}}$, by (ii) and (c) we have $N(B) \subset R\left[\left(\lambda_{k}-A\right)^{m_{k}}\right]$. Hence if $R[P(A)]$ is closed, then $B^{-1}(R[P(A)])=R\left[\left(\lambda_{k}-A\right)^{m_{k}}\right]+N(B)=R\left[\left(\lambda_{k}-\right.\right.$ $\left.-A)^{m_{k}}\right]$ is closed; and as $\left(\lambda_{i}-A\right)^{m_{i}}$ are commuting operators, we prove in the same form that they all have closed range. The final result follows from (ii) taking account that a continuous operator $B \in L(X)$ has dense range if and only if the conjugate $B^{\prime}$ is injective [2].

\section{Remarks}

(1) The above theorem, of course, can be obtained from the spectral mapping theorem for the Taylor spectrum associated to several commuting operators [3], but our version of the proof totally avoids Liouville's theorem in several complex variables, and in this sense is elementary.

(2) It is evident that the above corollary admits some generalizations: 
Parts (i) and (ii) can be extended to linear operators with domain $D(A) \neq X$ such that there exist $\alpha \in \mathrm{C}$ with $\alpha-A$ bijective. It suffices to consider factors of the form $(\lambda-A)^{k}(\alpha-A)^{-k}$, as the operator $P(A)$ has the same range and null space that $P(A)(\alpha-A)^{-n}$, where $n$ is the grade of the polynomial $P(z)$.

Analogously part (iii) can be extended to closed operators with nonvoid resolvent set. Note that in this case, by the closed graph theorem, $(\lambda-A)^{k}(\alpha-A)^{-k}$ is continuous.

\section{References}

1. H.A. GINDLER, A spectral mapping theorem for polynomials, Amer, Math. Monthly 72 (1965), 528-530.

2. A.E. TAYLOR, D.C. LAY, "Introduction to functional analysis," 2nd. ed., Wiley, 1980.

3. J.L. TAYLOR, The analytic functional calculus for several commuting operators, Acta Math. 125 (1970), 1-38.

4. T. YAMAMOTO, A note on the spectral mapping theorem, Siam J. Math. Anal. 2 (1971), 49-51.

\footnotetext{
Departamento de Matematicas

Facultad de Ciencias

39005 Santander, SPAIN.
}

Rebut el 15 de Febrer de 1988 